Journal of Research in Interprofessional

Practice and

Education

Vol. 6.1

June 2016

\title{
Do Interprofessional Educational Programs on Eating Disorders Provide Proximal and Distal Benefits? Findings from a National Cohort Collected from 1998 to 2010.
}

\author{
Jan H. Rosenvinge, PhD, \& Gunn Pettersen, PhD
}

\begin{abstract}
Background: Many programs are launched aiming to raise knowledge and competence in treating eating disorders, yet few of them have been evaluated.

Methods and Findings: Using a pre-post and one-year repeated measures design we evaluated a 17-month interprofessional education program (Body and SelfEsteem) comprising a national cohort of participants $(n=845)$ enrolled from 1998 to 2010. The purpose of the program is to raise health professionals' 1) knowledge, 2) confidence, 3) clinical competence, and 4) to promote an understanding of how patient care can be organized in an interdisciplinary fashion. The program format consists of five to six one- to three-day seminars with plenary lectures, and four to six closed network groups. The detected changes in all four outcomes were unrelated to program-irrelevant covariates. Program satisfaction was high, and on par with initial expectations.

Conclusions: Limited by the fact that a randomized controlled design was impossible to use, a reasonable conclusion is that the program may have provided both immediate and longer-term benefits.
\end{abstract}

Keywords: Eating disorders; Interprofessional; Education programs

Journal of Research in Interprofessional Practice and Education (JRIPE)

Vol. 6.1

(C) 2016

Corresponding author: Jan H. Rosenvinge. Email: jan.rosenvinge@uit.no

\section{Introduction}

Psychological, medical, interpersonal, and sociocultural features define the multifaceted nature of etiological and maintaining factors of eating disorders. An interprofessional approach to treatment is required to accommodate this nature. Barriers to implementing such an approach include a lack of knowledge about this nature of eating disorders, professional hierarchies favouring a particular profession's understanding [1], or an individual rather than a systemic approach to the raising of clinical competence and confidence [2,3].

Knowledge about the nature of eating disorders varies among health professionals. Deficits in knowledge and pejorative attitudes toward eating disorders and eating disorder patients have been found among primary care physicians $[4,5]$, obstetricians and gynaecologists [6], hospital physicians [7], and psychiatrists [8]. There are some inconsistent findings as to whether deficits in knowledge are unrelated [4] or related [9] to negative attitudes. Nevertheless, poor knowledge and negative attitudes 
2

Benefits from an Interprofessional Education Program

Rosenvinge \& Pettersen

Journal of Research in Interprofessional Practice and Education

Vol. 6.1

June 2016 may either separately or in combination explain why eating disorders are ranked poorly in terms of prestige among hospital doctors, primary care physicians, and medical students [10], as well as in the general population [9,11]. Both knowledge and attitudes have an impact on clinical services and clinical actions [4]. Negative attitudes and knowledge deficits are probably more prevalent in general than in specialized eating disorder clinics. As most eating disorder patients are treated in general clinics, this would imply that the majority of patients stand the risk of being exposed to clinicians providing suboptimal treatment services. This scenario calls for efforts to implement educational programs and to test whether they can promote positive attitudes and raise knowledge, confidence, and clinical competence. Further, programs that recruit participants sharing the same workplace in order to facilitate a systemic rather than an individual approach to improving such competence and confidence are called for.

In the last 30 years or so, the number of clinical courses and programs with an interprofessional focus has increased exponentially, mirroring a growing interest among students and professionals in taking part in such programs [12]. Recent systematic reviews $[12,13]$ indicate that such programs are well received by learners and that despite some methodological variability, tempering firm conclusions, such programs may raise knowledge and clinical competence and thus, contribute to improving the quality of treatment services.

Further, for eating disorders, there is a number of educational programs available. Some offer web-based programs aiming to increase clinical skills in a particular treatment approach, notably in cognitive-behavioural therapy [14,15]. Satisfaction with this approach seems related to the opportunity to illustrate clinical procedures and segments of treatment manuals [16]. Other kinds of programs have a more general aim of increasing knowledge about the nature of eating disorders, various treatment approaches, and the understanding of the need for an interprofessional treatment approach.

A pioneer interprofessional program [17] to enhance clinical competence in eating disorders was developed in Norway. This program was well received and some local spin-off benefits were identified, but like most programs, no formal evaluation was conducted. The most reliable form of evaluation to discern effects is, of course, the randomized controlled design. To our knowledge, only one study [18] has used such a design. Here, it was found that a theory-driven program for oral healthcare providers produced statistically significant improvements in general and procedural knowledge about the treatment of eating disorders as well as in more positive attitudes toward eating disorder patients. Self-selection to educational programs may preclude randomization, and thus, other studies have used uncontrolled designs. For instance, Linville, Aoyama, Knoble, and Gau [19] found increased knowledge and skills for addressing eating disorders after 45 health professionals had participated in a brief training program. Positive results were sustained at a short-term follow-up of six months. Moreover, Rosenberg (20) evaluated a one-day seminar targeting health professionals working in schools, which also showed significant changes in knowledge and attitudes. However, a high proportion of participants intending to work 
3

Benefits from an Interprofessional Education Program

Rosenvinge \& Pettersen

Journal of Research in Interprofessional Practice and Education

Vol. 6.1

June 2016 interprofessionally at the outset remained unchanged. These findings were flawed by short-term follow-ups, and up to 73 percent dropout from small sample sizes (i.e., $<50$ ), resulting in a substantial loss of statistical power and a lack of opportunity to perform multivariate analyses.

When a randomized controlled design is not feasible, multivariate analyses may allow for a study of covariates, which may indicate possible mechanisms. Hence, when controlling for initial values, program-relevant covariates explaining variance in outcome variables may support a hypothesis that a program may be beneficial. Conversely, an impact of program-irrelevant covariates such as clinical experience in treating eating disorders, age, or participants' own eating problems may not support the hypothesis. A study [1] where some covariates were controlled reported on findings from the first evaluation of an interprofessional educational program (Body and Self-Esteem). Here, the 78 participants' overall satisfaction with the program was generally good, particularly with respect to gaining theoretical knowledge, and understanding of eating disorders and gaining clinical skills.

The present study expands on this preliminary report by investigating the full national cohort of participants from all the years (i.e., 1998-2010) this program was run, and exploring the following research questions:

- Do participants report being more clinically confident in treating eating disorder patients after completing the program?

- Do participants report that the program facilitated an interest in interprofessional work?

- Do participants increase their knowledge about eating disorders and change their professional attitudes toward understanding and treating eating disorder patients after finishing the program?

- What is the level of overall and sub-domain program satisfaction relative to initial expectations about the benefits of the program?

- What is the role of content-relevant covariates in explaining changes in the independent outcome variables "clinical confidence," "interprofessional work," and "overall satisfaction"?

Our expectations are that participants report a facilitation of interprofessional work, higher confidence, increased knowledge, and more positive attitudes toward treatment after completing the program. In addition, we expect a high level of immediate and long-term program satisfaction and that initial expectations are met. Well aware of the inability to discern causal effects, we expect that for the final question the variation in the independent outcome variables is explained by covariates related to the program content and purpose, and not by content-irrelevant covariates, i.e., age, gender, number of treated eating disordered patients at pretest, self-reported eating disturbances, and level of specialized clinical experience.

\section{The educational program: Body and self-esteem}

The overall purpose of the Body and Self-Esteem program is to raise health professionals' knowledge, confidence, and clinical competence in eating disorders and to 
4

Benefits from an Interprofessional Education Program

Rosenvinge \& Pettersen
Journal of Research in Interprofessional Practice and Education

Vol. 6.1

June 2016 promote an understanding of how patient care can be organized in an interdisciplinary manner. From a longer-term perspective, a goal of the program is to encourage participants to treat more eating disorder patients. Another goal is to inspire participants to act as resource individuals in the field of eating disorders in their local communities or treatment centres in terms of education, clinical supervision, and as the founders of professional network groups.

The program has been funded by national health authorities, and disseminated nationally through all the four national health regions. In every region, a part-time paid regional coordinator is responsible for program implementation.

Within the total timeframe of one-and-a-half-years for each program wave, the format consists of five to six one- to three-day seminars with plenary lectures, and four to six closed network groups. Plenary lectures are given by leading national and international experts as well as by representatives from patient organizations. The lectures focus on empirically based models of understanding eating disorders, medical complications, diagnostic and general clinical evaluation, prevention, comorbidity, evidence-based treatment models, and long-term outcome. In addition, participants receive a booklet of updated literature before and during the lectures. Each network group consists of five to seven participants headed by an experienced clinician, who acts as a supervisor. All groups meet for 50 hours during the program course, and each participant should present and receive feedback on the clinical work with at least one to two eating disorder cases from their portfolio. The groups are composed of people from various health professions and within the same geographical area or workplace to facilitate interprofessional learning and a systemic approach to longerterm networking.

\section{Methods}

\section{Subjects}

In total, 845 clinicians (mean age 43.60 years [SD 8.64, range 20-68]) enrolled in the program from 1998 to 2010 . Of these 8 percent $(n=68)$ were men, 89.5 percent $(n=756)$ were women, and 2.5 percent $(n=22)$ failed to report their gender. The majority, 43.2 percent $(n=365)$ were registered nurses, 20.8 percent $(n=176)$ were clinical psychologists, 7.6 percent $(n=64)$ were medical doctors, 18.9 percent $(n=160)$ had other health professions, 7.0 percent $(n=59)$ were not health professionals, and 2.5 percent $(n=21)$ failed to report any profession. The majority $(60 \%$, $n=507)$ worked in specialist healthcare, 25 percent $(n=154)$ in primary care settings, and the remaining 15 percent either worked in other settings or failed to report any workplace.

\section{Measures}

We used 12 statements about clinical confidence (Table 1) and 22 statements (Table 2) to measure knowledge about eating disorders. The latter intended to discern intuitively true or false responses from true or false responses based on actual knowledge about eating disorders. Moreover, 21 statements covered professional attitudes about the treatment of eating disorders (Table 3). 
Table 1. Main effects for changes in scores on statements about clinical confidence using a 1-10-point scale (10 = maximal agreement). The highest effect sizes $\left(\eta^{2}\right)$ are printed in bold.

\begin{tabular}{|c|c|c|c|c|c|c|c|c|c|c|}
\hline & \multirow[t]{2}{*}{ N } & \multicolumn{2}{|c|}{ Pretest } & \multicolumn{2}{|c|}{ Posttest } & \multicolumn{2}{|c|}{ One-year follow-up } & \multirow[t]{2}{*}{$\mathrm{F}$} & \multirow[t]{2}{*}{ p } & \multirow[t]{2}{*}{$\eta^{2}$} \\
\hline & & M & SD & M & SD & M & SD & & & \\
\hline I see myself as a local resource person in the field of eating disorders. & 135 & 4.91 & 1.96 & 6.16 & 2.61 & 8.00 & 2.12 & 82.69 & 0.0001 & 0.38 \\
\hline Others believe me to be a local resource person in the field of eating disorders. & 99 & 4.97 & 2.14 & 6.84 & 2.27 & 7.85 & 2.22 & 57.84 & 0.0001 & 0.37 \\
\hline I have given, or plan to give, lectures on eating disorders. & 132 & 5.26 & 2.35 & 6.49 & 2.78 & 5.28 & 3.28 & 12.03 & 0.0001 & 0.08 \\
\hline I tend not to treat eating disorder patients to avoid a difficult clinical problem. & 115 & 3.50 & 2.22 & 3.14 & 2.78 & 2.30 & 1.86 & 9.88 & 0.0001 & 0.08 \\
\hline I tend not to treat eating disorder patients to provide them better help elsewhere. & 131 & 6.79 & 2.54 & 7.31 & 3.12 & 6.63 & 3.71 & 16.94 & 0.11 & 0.02 \\
\hline I tend not to treat eating disorder patients because I do not have sufficient knowledge to do so. & 129 & 5.16 & 2.64 & 3.28 & 2.78 & 4.11 & 3.07 & 21.39 & 0.0001 & 0.14 \\
\hline I feel confident in treating eating disorder patients if I can follow a treatment manual. & 116 & 5.00 & 2.27 & 4.81 & 3.06 & 4.09 & 2.59 & 5.04 & 0.01 & 0.04 \\
\hline I feel confident in treating eating disorder patients if I can receive clinical supervision. & 96 & 7.14 & 2.11 & 8.06 & 2.07 & 8.83 & 2.89 & 7.59 & 0.001 & 0.07 \\
\hline I feel confident in treating eating disorder patients if I can collaborate with other kinds of health professionals. & 99 & 7.48 & 2.18 & 8.47 & 1.81 & 8.01 & 2.43 & 5.67 & 0.004 & 0.06 \\
\hline I feel confident in treating eating disorder patients when I can see that the patient's condition improves. & 99 & 7.48 & 1.84 & 7.96 & 1.84 & 7.77 & 2.39 & 1.67 & 1.67 & 0.02 \\
\hline I feel confident in treating eating disorder patients because I have sufficient theoretical knowledge about eating disorders. & 99 & 6.31 & 2.41 & 7.71 & 2.04 & 8.10 & 2.21 & 21.94 & 0.0001 & 0.18 \\
\hline I feel confident in treating eating disorder patients because I see many such patients. & 99 & 4.73 & 2.48 & 5.27 & 2.90 & 5.70 & 3.05 & 5.65 & 0.001 & 0.06 \\
\hline
\end{tabular}

Note: $\mathrm{N}=$ number of cases, $\mathrm{M}=$ mean score, $\mathrm{SD}=$ standard deviation, $\mathrm{F}=$ an overall ratio for the test of differences, $\mathrm{p}=$ probability of rejecting the null hypothesis, $\mathrm{n} 2=$ the effect size 
Table 2. Score changes on items covering statements about eating disorders. All items range from $1-10$ with 10 ( $10=$ maximal agreement). All comparisons are calculated using a paired $t$-test. Medium to large effect sizes (Hedges' $g \geq 0.50$ ) are printed in bold. Items are marked as true (T) or false (F) based on research and general consensus in the field of eating disorders.

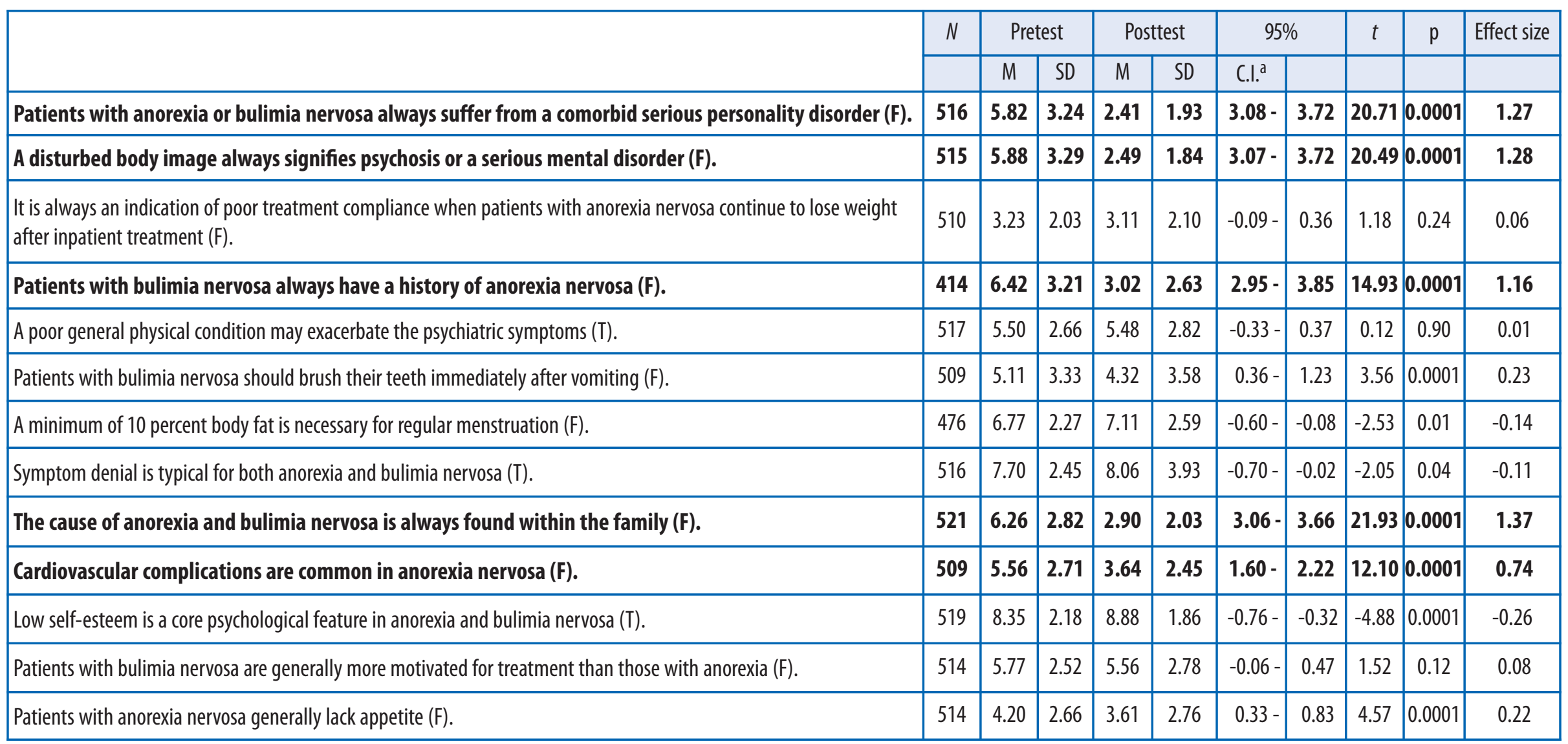


Table 2. Score changes on items covering statements about eating disorders. (continued)

\begin{tabular}{|c|c|c|c|c|c|c|c|c|c|c|}
\hline & \multirow[t]{2}{*}{$N$} & \multicolumn{2}{|c|}{ Pretest } & \multicolumn{2}{|c|}{ Posttest } & \multicolumn{2}{|c|}{$95 \%$} & \multirow[t]{2}{*}{$t$} & \multirow[t]{2}{*}{$\mathrm{p}$} & \multirow[t]{2}{*}{ Effect size } \\
\hline & & M & SD & M & SD & C.I. ${ }^{\mathrm{a}}$ & & & & \\
\hline Patients with anorexia nervosa generally lack appetite (F). & 514 & 4.20 & 2.66 & 3.61 & 2.76 & $0.33-$ & 0.83 & 4.57 & 0.0001 & 0.22 \\
\hline It is always a sign of improvement if patients with eating disorders become pregnant (F). & 411 & 6.25 & 2.47 & 4.18 & 2.47 & $1.88-$ & 2.60 & 12.34 & 0.0001 & 0.84 \\
\hline It is always a good sign when the eating disorder patient reports on treatment progress (F). & 519 & 5.86 & 2.38 & 4.43 & 2.43 & $1.11-$ & 1.74 & 9.01 & 0.0001 & 0.60 \\
\hline Most young people who diet develop an eating disorder (F). & 339 & 2.31 & 1.68 & 2.09 & 3.64 & $-0.20-$ & 0.62 & 1.01 & 0.31 & 0.07 \\
\hline Eating disorders are most prevalent in the middle class (F). & 332 & 2.81 & 2.25 & 2.61 & 2.26 & $-0.08-$ & 0.48 & 1.41 & 0.16 & 0.09 \\
\hline People with anorexia nervosa rarely set too ambitious self-demands in life (F). & 440 & 2.28 & 1.65 & 1.84 & 1.58 & $0.22-$ & 0.66 & 4.07 & 0.0001 & 0.64 \\
\hline Most young people with anorexia nervosa starve themselves to death (F). & 329 & 2.90 & 2.23 & 2.00 & 1.60 & $0.62-$ & 1.17 & 6.93 & 0.0001 & 0.45 \\
\hline Bulimia nervosa is a consequence of a failure of structuring one's daily routines (F). & 440 & 2.31 & 1.68 & 1.85 & 1.63 & $0.27-$ & 0.66 & 4.75 & 0.0001 & 0.28 \\
\hline Patients with bulimia are more perfectionistic than those with anorexia nervosa (F). & 336 & 2.29 & 1.65 & 1.85 & 1.58 & $-0.52-$ & 0.03 & 4.07 & 0.0001 & 0.64 \\
\hline Most people with anorexia nervosa are cured within a couple of months (F). & 340 & 2.12 & 1.72 & 1.94 & 1.73 & $0.06-$ & 0.42 & 1.47 & 0.14 & 0.11 \\
\hline
\end{tabular}

Note: The superscript "a" indicates the 95 percent confidence interval (Cl) for the difference. 
Table 3. Score changes on items covering professional attitudes toward treating eating disorder patients. All items range from 1-10 (10 = maximal agreement). All comparisons are calculated using a paired t-test. Medium and large effect sizes (Hedges' $g \geq 0.50$ ) are printed in bold. Some items are marked as true $(T)$ or false $(F)$ based on research and consensus in the field of eating disorders.

\begin{tabular}{|c|c|c|c|c|c|c|c|c|c|c|}
\hline & \multirow[t]{2}{*}{$N$} & \multicolumn{2}{|c|}{ Pretest } & \multicolumn{2}{|c|}{ Posttest } & \multicolumn{2}{|c|}{$95 \%$} & \multirow[t]{2}{*}{$t$} & \multirow[t]{2}{*}{$p$} & \multirow[t]{2}{*}{$g$} \\
\hline & & M & SD & M & SD & C.I. ${ }^{\mathrm{a}}$ & & & & \\
\hline The main recovery criteria for anorexia nervosa is regular menstruation and normal weight for at least 6 months. & 517 & 5.46 & 2.51 & 5.32 & 2.78 & $-0.12-$ & 0.39 & 1.06 & 0.29 & 0.05 \\
\hline It is unrealistic to think that a particular treatment is suitable for all eating disorder patients. & 343 & 7.53 & 2.57 & 8.14 & 2.48 & $-.88--$ & 0.34 & -4.45 & 0.0001 & 0.24 \\
\hline Treatment works better if the patient is informed about the risk of medical complications. & 539 & 5.33 & 2.40 & 5.40 & 2.60 & $-0.33-$ & 0.18 & -0.59 & 0.55 & 0.03 \\
\hline Female therapists generally create a better therapeutic alliance to eating disorder patients. & 544 & 4.08 & 2.61 & 3.50 & 2.71 & $0.32-$ & 0.84 & 4.43 & 0.0001 & 0.29 \\
\hline Control and surveillance after meals is a core procedure of inpatient treatment. & 537 & 6.61 & 2.63 & 7.14 & 2.66 & $-0.81--$ & 0.23 & -3.64 & 0.0001 & 0.20 \\
\hline Insight into underlying psychological problems makes eating disorder symptoms disappear. & 542 & 3.57 & 2.47 & 3.29 & 2.45 & $0.01-$ & 0.55 & 2.01 & 0.05 & 0.11 \\
\hline Diagnostic evaluation is seldom necessary to treat eating disorder patients. & 542 & 3.58 & 2.79 & 2.95 & 2.73 & $0.35-$ & 0.92 & 4.33 & 0.0001 & 0.23 \\
\hline Interprofessional collaboration blurs the medical responsibility. & 545 & 2.88 & 2.51 & 2.25 & 2.23 & $0.35-$ & 0.90 & 4.50 & 0.0001 & 0.15 \\
\hline Treatment of eating disorder patients is highly complicated by patients' poor motivation and manipulative behaviours. & 544 & 6.14 & 2.86 & 5.96 & 3.12 & $-0.12-$ & 0.48 & 1.19 & 0.24 & 0.06 \\
\hline Eating disorder symptoms disappear when the whole family gets help. & 543 & 3.19 & 2.32 & 2.76 & 2.19 & $-0.18-$ & 0.70 & 3.30 & 0.001 & 0.17 \\
\hline Standardized instruments disturb the therapeutic relation. & 517 & 4.13 & 2.34 & 3.48 & 2.35 & $0.42-$ & 0.89 & 5.41 & 0.0001 & 0.55 \\
\hline The main message to eating disorder patients is that they should eat more or more regularly. & 466 & 3.90 & 2.50 & 3.62 & 2.60 & $-0.01-$ & 0.56 & 1.90 & 0.06 & 0.11 \\
\hline A major and serious weight loss requires inpatient hospital treatment (T). & 509 & 5.51 & 2.62 & 5.51 & 2.80 & $0.0-$ & 0.90 & 0.00 & 0.97 & 0.00 \\
\hline
\end{tabular}


Table 3. Score changes on items covering professional attitudes toward treating eating disorder patients. (Continued)

\begin{tabular}{|c|c|c|c|c|c|c|c|c|c|c|}
\hline & \multirow[t]{2}{*}{$N$} & \multicolumn{2}{|c|}{ Pretest } & \multicolumn{2}{|c|}{ Posttest } & \multicolumn{2}{|c|}{$95 \%$} & \multirow[t]{2}{*}{$t$} & \multirow[t]{2}{*}{$p$} & \multirow[t]{2}{*}{$g$} \\
\hline & & M & SD & M & SD & C.I. ${ }^{\mathrm{a}}$ & & & & \\
\hline Inpatient hospital treatment is suitable to make the patient realize the severity of her condition (F). & 519 & 5.77 & 2.54 & 4.11 & 2.23 & $1.35-$ & 1.97 & 10.61 & 0.0001 & 0.28 \\
\hline It is generally recommended to treat bulimia by inpatient hospital treatment $(F)$. & 511 & 5.89 & 2.94 & 3.04 & 1.98 & $2.54-$ & 3.17 & 17.60 & 0.0001 & 1.14 \\
\hline The faster one accomplishes weight gain in anorexia nervosa, the better (F). & 519 & 3.43 & 2.22 & 3.43 & 2.40 & $-0.23-$ & 0.23 & 0.00 & 0.99 & 0.00 \\
\hline Patients with bulimia should remain on antidepressants as long as possible (F). & 509 & 5.33 & 2.90 & 3.61 & 2.37 & $1.40-$ & 2.04 & 10.56 & 0.0001 & 0.65 \\
\hline Anorexia nervosa is not an indication to use anti-psychotic medication (T). & 511 & 5.17 & 3.32 & 7.59 & 3.00 & $-2.82-$ & -2.02 & -11.89 & 0.0001 & -0.76 \\
\hline Psychiatric treatment is useless as long as the medical condition is poor ( $T$ ). & 517 & 5.50 & 2.66 & 5.48 & 2.82 & $-0.33-$ & 0.37 & 0.12 & 0.90 & 0.00 \\
\hline Family treatment is usually more relevant when the patient is a teenager $(\mathrm{T})$. & 407 & 4.86 & 2.51 & 7.05 & 2.51 & $-2.56-$ & 8.83 & -11.85 & 0.0001 & 0.87 \\
\hline Systematic positive reinforcement of weight gain is an optimal treatment of anorexia nervosa (F). & 507 & 5.77 & 2.45 & 4.03 & 2.42 & $0.16-$ & 1.44 & -11.20 & 0.0001 & 0.71 \\
\hline
\end{tabular}

Note: The superscript "a" indicates the 95 percent confidence interval (CI) for the difference.

Pretest program expectations and satisfaction (posttest and one-year follow-up) were measured as a total score, and in terms of the four subdomains "theoretical knowledge," "practical skills," "personal development," and "coherence between theory and clinical practice." Further, they rated satisfaction with the lecture themes and the group supervision activity (Table 4). Moreover, at all three measurement points, the participants rated their clinical experience in eating disorders besides reporting the number of eating disorder patients they had treated during the past 12 months. In addition, they completed a self-report measure of eating disorder problems, i.e., the Eating Disturbance Scale (EDS-5) [21], and responded to a statement about their involvement in interprofessional networks. 
Table 4. Mean scores (SD) on statements about program expectations and satisfaction, facilitation of interprofessional work, and satisfaction with specific parts of the overall program. All items are scored 1-10

(10 = maximal agreement). Effect sizes (Hedges' $g \geq 0.50$ ) are printed in bold.

\begin{tabular}{|c|c|c|c|c|c|c|c|c|}
\hline & \multicolumn{2}{|c|}{ Pretest expectation } & \multicolumn{2}{|c|}{ Posttest satisfaction } & \multicolumn{2}{|c|}{ One-year satisfaction } & \multirow[t]{2}{*}{$p$} & \multirow{2}{*}{$\begin{array}{c}\text { Effect size } \\
\text { (Hedges' } g \text { ) }\end{array}$} \\
\hline & M & SD & M & SD & M & SD & & \\
\hline Overall satisfaction score $(n=551)$ & 9.71 & 0.64 & 8.71 & $1.55^{\mathrm{a}}$ & 9.12 & 2.96 & & \\
\hline Increased theoretical knowledge $(n=670)$ & 8.47 & 1.72 & 8.25 & 1.60 & 8.58 & $2.20^{\mathrm{b}}$ & & \\
\hline Increased practical skills $(n=669)$ & 8.27 & 1.83 & 7.48 & $2.08^{c}$ & 8.16 & $2.20 \mathrm{~d}$ & & \\
\hline Increased personal development $(n=667)$ & 8.27 & 1.85 & 7.78 & $2.00^{e}$ & - & - & & \\
\hline Increased understanding about how theory and clinical practice relate $(n=665)$ & 8.83 & 1.31 & 8.04 & $1.88^{f}$ & 8.21 & $2.09 \mathrm{~g}, \mathrm{~h}$ & & \\
\hline Facilitation of interprofessional work $(n=495)$ & 8.20 & 2.04 & 8.43 & 1.98 & 8.08 & 3.25 & & \\
\hline Group supervision $(n=231)$ & - & - & 9.13 & 1.63 & 7.95 & 2.01 & 0.0001 & 0.64 \\
\hline Plenary lectures (overall mean score) $(n=414)$ & - & - & 7.79 & 0.86 & 7.21 & 1.48 & 0.0001 & 0.48 \\
\hline Epidemiology $(n=499)$ & - & - & 7.35 & 1.57 & 7.09 & 2.13 & n.s. & 0.00 \\
\hline Approaching the patient $(n=527)$ & - & - & 8.21 & 1.51 & 8.00 & 1.93 & n.s. & 0.00 \\
\hline Family therapy $(n=481)$ & - & - & 7.95 & 1.55 & 6.67 & 2.73 & 0.0001 & 0.58 \\
\hline Diagnostic issues $(n=454)$ & - & - & 6.67 & 1.58 & 7.54 & 2.07 & 0.01 & 0.47 \\
\hline Theoretical lectures $(n=417)$ & - & - & 9.16 & 1.11 & 7.84 & 1.82 & 0.0001 & 0.82 \\
\hline Group treatment $(n=473)$ & - & - & 6.54 & 2.28 & 5.82 & 2.66 & 0.0001 & 0.29 \\
\hline How to work interprofessionally $(n=495)$ & - & - & 8.46 & 1.58 & 7.66 & 2.25 & n.s. & 0.00 \\
\hline Prevention $(n=526)$ & - & - & 7.43 & 2.58 & 6.97 & 2.54 & 0.0001 & 0.18 \\
\hline Comorbidity $(n=499)$ & - & - & 8.56 & 1.48 & 7.77 & 2.00 & n.s. & 0.00 \\
\hline Individual treatment $(n=523)$ & - & - & 8.75 & 1.53 & 8.35 & 1.58 & 0.0001 & 0.26 \\
\hline Hospital treatment $(n=414)$ & - & - & 7.83 & 2.26 & 6.24 & 2.41 & n.s. & 0.00 \\
\hline Medical examination $(n=531)$ & - & - & 6.84 & 1.57 & 7.30 & 2.33 & 0.0001 & 0.23 \\
\hline
\end{tabular}

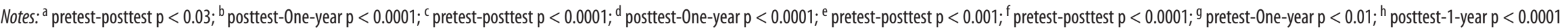
(pairwise t-tests; all effect sizes range $0.09 \mathrm{~h}-0.51 \mathrm{a}),-=$ not measured.

Journal of Research in Interprofessional

Practice and Education 
11

Benefits from an Interprofessional Education Program

Rosenvinge \& Pettersen
Journal of Research in Interprofessional Practice and Education

Vol. 6.1

June 2016
All measures had a response format of 1 to 10 , with 10 as the most favourable or optimal score, except for the EDS-5, where 10 indicated the most disturbed eating problems.

\section{Design and procedure}

We used a repeated measures design, and data were collected at the first (pretest) and the final (posttest) sessions of the program, as monitored by the program administrators. One-year follow up measures were mailed to the participants, and to reduce dropouts, they only completed measures of confidence and program satisfaction.

\section{Statistical analyses}

Data were analyzed with bivariate comparisons using pairwise $t$-tests, repeated measures analysis of covariance (ANCOVA), and stepwise multiple regression procedures. In the latter procedure, we tested the following independent outcome variables: the total satisfaction score, clinical confidence measures, which changed the most according to effect sizes, and the experienced facilitation of interprofessional work at the posttest and the one-year analyses. At step one, the program-unrelated variables were entered (i.e., pretest measures of age, self-reported eating problems, clinical experience, and number of treated eating disorder patients). At step two, we entered the program-related covariates (i.e., items achieving at least medium effect sizes $(\geq 0.50)$ covering knowledge and attitudes toward eating disorder at the posttest and the one-year follow-up). In step three, we entered the subdomain satisfaction scores, and in step four, we controlled for program expectations.

\section{Results}

On a 10-point scale (maximum score $=10$ ), participants scored 3.90 (SD 1.77, range 1-9) and 3.97 (SD 2.03, range 1-9) on the pretest self-rated overall measures of knowledge and clinical experience with eating disorders, respectively. The mean number of eating disorder patients they had treated the past 12 months prior to the pretest was 4.70 (SD 6.11, range 0-60).

\section{Stability and change in clinical confidence and interest in interprofessional work}

As for confidence, at the one-year follow-up, the participants viewed themselves and believed others to view them as local resource persons in the field of eating disorders, and due to an experience of increased knowledge, they felt more confident in treating eating disorder patients. Hence, they also disagreed with the statement that they declined to treat eating disorder patients to avoid a clinical problem; however, the significant decrease in the agreement score yielded a low effect size (i.e., 0.08; see Table 1). Moreover, the participants tended to agree more with the statement that interprofessional collaboration increased their confidence in treating eating disorder patients (see Table 1). These findings correspond with a statistically significant $(\mathrm{p}<0.0001)$ decreasing trend to disagree with the statement that interprofessional collaboration blurs medical responsibility, although the effect size was low (i.e., 0.15; Table 4). These 
12

Benefits from an Interprofessional Education Program

Rosenvinge \& Pettersen

Journal of Research in Interprofessional Practice and Education

Vol. 6.1

June 2016 experienced contextual benefits may be balanced against the fact that the mean number of eating disorder patients treated over the past 12 months increased non-significantly from 4.7 (SD 6.11; pretest) to 5.3 (SD 7.04; posttest) and to 6.0 (SD 3.36; 1 year).

\section{Stability and change in knowledge and professional attitudes toward eating disorders}

Changes in knowledge about eating disorders from pretest to posttest are displayed in Table 2. After finishing the program, participants agreed less to the following objectively false statements: a) that eating disorder patients always suffer from a severe comorbid personality disorder ( $p<0.0001$, effect size [ES] 1.27), b) that a disturbed body image indicates a psychosis ( $p<0.0001$; ES 1.28), c) that people with bulimia always have a history of anorexia nervosa $(p<0.0001$; ES 1.16), d) that the cause of eating disorders are always found within the family ( $p<0.0001$; ES 1.37), e) that cardiovascular complications are common in anorexia nervosa ( $p<0.0001$; ES 0.74$)$, f) that pregnancy is always a sign of improvement ( $p<0.0001$; ES 0.60$)$, g) that people with anorexia nervosa rarely set too ambitious goals for themselves, and $h$ ) that bulimia patients are more perfectionistic than are those with anorexia nervosa $(p<0.0001$; ES 0.64).

Some professional attitudes toward treatment also changed significantly and with high effect sizes (see Table 3). Notably, participants were more positive regarding the use of standardized instruments ( $p<0.0001$; ES 0.55 ), and family-based treatment for teenage patients $(p<0.0001$; ES 0.87). They were more hesitant toward treating bulimia in hospital $(p<0.0001$; ES 1.14), toward long-term use of antidepressants $(p<0.0001$; ES 0.65), to use medication ( $p<0.0001$; ES 0.76), and to use positive reinforcement of weight gain in treating anorexia nervosa $(p<0.0001$; ES 0.71).

\section{Immediate and longer-term satisfaction}

Table 4 shows a high overall positive expectation when entering the program. It is noteworthy that despite a drop in overall satisfaction at the posttest, the one-year score is on par with the initial expectations. A similar pattern is found for the subdomain scores on "theoretical knowledge" and "practical skills," while the high expectations about understanding the relation between theory and clinical practice are not fully met at the posttest or at the one-year follow-up. The mean satisfaction score, comprising the 12 lecture themes during the seminars, was 7.79 (SD 0.86; posttest) and 7.21 (SD 1.48; one-year follow-up), respectively, and both reductions were statistically significant $(\mathrm{p}<0.0001$; ES 0.48$)$. Satisfaction with the clinical supervision part of the program was high at the posttest $(9.13$; SD 1.63) and also declined at the oneyear follow-up (7.95; SD 2.01; $p<0.0001$; ES 0.64) yet supervision surpassed the overall score for plenary lectures at both measurement points $(p<0.0001)$, but the effect size was higher at the posttest (i.e., 1.03) than it was at the one-year follow-up (i.e., 0.42). Moreover, the reported benefit from the program in terms of facilitation of interprofessional work was high at the posttest (8.43; SD 1.98) and remained unchanged $(8.08$; SD 3.25, $p<0.62)$ at the one-year follow-up. 
Benefits from an Interprofessional Education Program

Rosenvinge \& Pettersen

Journal of Research in Interprofessional Practice and Education

Vol. 6.1

June 2016

\section{Journal of Research in Interprofessional Practice and Education}

\section{Predictors of outcome variables}

\section{Confidence}

Using the three variables with the highest effect sizes (see Table 2) as predictors, participants who saw themselves as a local resource person also displayed high overall satisfaction with the program. They also tended to believe that others regarded them as such $(F=6.40 ; \mathrm{p}<0.004$, and $F=5.63 ; p<0.004)$, but the effect sizes were low ( 0.07 and 0.08 , respectively). No other program-related or unrelated variables could be entered. In the repeated measures ANCOVA, the overall time effect of confidence in terms of seeing oneself as a local resource person remained significant $(F=4.08 ; \mathrm{p}<0.02)$. However, there was a significant interaction with one non-program-related variable (i.e., self-reported clinical experience) at the pretest $(p<0.04)$, and the partial effect size (i.e., the proportion of explained variance not accounted for by the covariate) was low (i.e., 0.10). As for the belief that others viewed them as local resource persons, the main effect became non-significant $(F=2.66, P=0.11)$, and no interaction effects were detected. Higher levels of self-reported clinical experience in treating eating disorders explained clinical confidence due to sufficient knowledge (ES 0.18, see Table 2), but this was only significant at the pretest $(F=4.57, p<0.01)$, and the effect size was low (0.06). Again, no other programrelated or unrelated variables could be entered. In the repeated measures analysis, the main effect became non-significant $(F=2.85 ; p=0.07$, partial $\mathrm{ES}=0.08)$ when covariates were entered, yet no significant program-relevant or irrelevant covariate interactions were found.

\section{Facilitation of interprofessional work}

The final posttest model was not significant $(F=1.07 ; p<0.11)$ and accounted for only 2 percent of the variance (adjusted $\mathrm{R}^{2}$ ), and was explained by the satisfaction sub-domain "practical skills" (standardized beta $=0.13 ; \mathrm{p}<0.0001$ ). At the one-year follow-up, the experienced facilitation of interprofessional work (final model $F=$ $1.73 ; p<0.001)$ explained 3 percent of the variance, and only the satisfaction subdomain "personal benefit" (standardized beta $=0.10 ; p<0.01$ ) at the posttest could be entered. In the repeated measures analysis, the time change became non-significant when covariates were included, yet no interactions with program-relevant or irrelevant covariates were found.

\section{Overall program satisfaction}

At the posttest the final model $(F=26.70 ; p<0.0001)$ accounted for 34 percent of the variance in overall program satisfaction, and overall satisfied participants were also satisfied with the program in terms of providing more theoretical knowledge (standardized beta $=0.30 ; p<0.0001$ ), and a better understanding of how theory and clinical work are related (standardized beta $=0.37 ; p<0.0001$ ). Nevertheless, none of those items covering knowledge and attitudes and showing the largest change in scores over time (i.e., with ES $\geq 0.50$, tables 2 and 3, respectively) could be entered, as was also the case for program-unrelated variables. At the one-year follow-up, the final model $(F=79.44, p<0.0001)$ only entered theoretical benefit to explain the 50 
Benefits from an Interprofessional Education Program

Rosenvinge \& Pettersen

Journal of Research in Interprofessional Practice and Education

Vol. 6.1

June 2016 percent of variation in overall satisfaction, but again, none of the items covering attitudes or knowledge could be entered. Significant correlations were found between overall program satisfaction and satisfaction with group supervision $(r=0.18$; $p<$ $0.05)$ and plenary lectures $(r=0.29 ; p<0.01)$, respectively. However, such evaluations may be highly influenced by factors remotely relevant for the program purpose and content, as indicated by non-significant partial correlations when sub-domain satisfaction scores were controlled for. The repeated measures analysis revealed no time effect when covariates were included, and no program-relevant or irrelevant covariate interactions were detected.

\section{Attrition analyses}

As not all participants responded to each of the items at all measure points we analyzed differences in mean score between those who did in comparison with the full sample. On the items covering knowledge about eating disorders and on the items covering attitudes toward treatment, and where a time effect was shown with an effect size $\geq 0.50$ (see Tables 3 and 4), no statistical differences were found, and the effect sizes were marginal (i.e., $\leq 0.11$ ).

\section{Discussion}

The first and second aims of our study were to search for changes in terms of clinical confidence, and whether the participants reported that interprofessional work increased their confidence. Table 1 shows that changes did occur, and that interprofessional collaboration significantly raised the participants' clinical confidence, yet the effect size was small. Notably, after one year, there was a markedly higher agreement with statements that they believed themselves and others to view them as local resource persons in the field of eating disorders. Moreover, they agreed more with the statement that they felt confident in treating eating disorder patients due to increased knowledge about eating disorders. Hence, they disagreed more with the statement that they tended not to treat eating disorder patients to avoid a clinical problem (see Table 1). Still, they did not report treating significantly more eating disorder patients in this time period. However, such a discrepancy can be accounted for by fluctuating trends in admission rates. In sum, then, our expectations were met with respect to increased confidence and interest in interprofessional work. These findings concur with qualitative analyses from another study [22].

The third aim was to search for change versus stability in professional attitudes toward understanding and treating eating disorder patients, as well as in knowledge about eating disorders. Notably, at the posttest, participants tended to disagree more with statements favouring that all eating disordered patients suffer from a serious mental disorder, that their body distortions signify a psychosis, that those with bulimia nervosa always have a history of anorexia, and that the family is the cause of eating disorders. Moreover, they changed their views about perfectionism and eating disorders and about aspects of recovery. Change was also observed for attitudes toward treatment. Notably, there was more positive opinion about using standardized instruments to measure current clinical status and treatment progress, less belief 
15

Benefits from an Interprofessional Education Program

Rosenvinge \& Pettersen

Journal of Research in Interprofessional Practice and Education

Vol. 6.1

June 2016 in using systematic positive reinforcement as an approach to weight restoration, and approaching more evidence-based use of medications (see Table 3). Similar changes in knowledge and attitudes have been noted in previous studies [1,19]. Only nine of twenty-two knowledge items (see Table 2) and six of twenty-one attitudinal items (see Table 3) reached large effect sizes. Such low proportions could be related to high pretest values, indicative of good initial knowledge and a possible ceiling effect because of a self-selection bias. However, our expectations of change are met in the sense that the strong effect sizes indicate a considerable change in knowledge and attitudes during and after the program.

As for the fourth aim, most previous programs show high consumer satisfaction [12], and our findings are no exception, thus supporting our initial expectations. Contrary to most other studies, we were also able to compare with initial expectations to control for the positive biases in self-selection to the program. Also contrary to most studies, our follow-up period was considerably longer, thus evading the risk of positively inflated immediate scores because of a personal investment in finishing the program. Finally, considering the fact that overall, and throughout the 12-year data collection period, the mean dropout during the program was below two percent, our findings indicate that the participants were highly satisfied after completion and at the one-year follow-up. In fact, the one-year findings were somewhat better than the posttest data for overall satisfaction, satisfaction in terms of practical skills, understanding the relationship between theory and clinical practice, and increased theoretical knowledge (see Table 4). The latter should be balanced against the fact that satisfaction with the theoretical lectures dropped 1.32 points to $7.84(p<0.0001$; ES 0.82). An additional eight scores for specific parts of the lecture content also dropped, but on the other hand, such specific findings may appear because of decay in memory recall.

Another finding was that the participants' high expectations in terms of learning to work professionally were generally met. This finding concurs with our previous qualitative investigation [22] of the one-year impact of this program. Here, an impetus toward establishing interprofessional clinical teams, and to contribute to a better organization of the healthcare for eating disorder patients was clearly expressed. Highly positive attitudes toward interprofessional work also concur with other previous findings [20]. These findings should be tempered against possible self-selection effects in terms of a higher probability for enrollment in an interprofessional education program for professionals who already value and appreciate interprofessional work. The relevance of this bias is supported by the rather low agreement at all measurement points with the negative attitudes toward interprofessional collaboration (see Table 3).

Our final aim was to examine the relationship between outcome variables and content-relevant covariates. Participants' improved confidence in terms of acting as local resource persons may appear as a non-specific benefit in the sense that it was related to overall program satisfaction, but not to any specific changes in knowledge or attitudes. The participants reported more confidence due to experiencing more knowledge, but the notion of non-specific program benefits is supported by the fact that 
16

Benefits from an Interprofessional Education Program

Rosenvinge \& Pettersen

Journal of Research in Interprofessional Practice and Education

Vol. 6.1

June 2016 none of the items measuring knowledge contributed in explaining the variance. Alternatively, by participating in the program, the participants may have gained more knowledge and acquired attitudes toward treatment, but such changes were not captured by our choice of knowledge and attitudinal variables. Nevertheless, the changes in confidence indeed mirrored the overall purpose of the Body and Self-Esteem program. A similar kind of argument is valid for the outcome variable "facilitating interprofessional work," and for predictors of overall program satisfaction. For instance, program satisfaction was actually related to a theoretical benefit at the one-year follow-up, but our selection of items covering knowledge could not be entered in the multivariate analyses. A positive attitude toward working interprofessionally remained throughout. This outcome variable was only marginally explained by some satisfaction sub-domain scores, but again, not related to variables measuring knowledge and attitudes.

In sum then, the present program may appear as beneficial in terms of confidence, understanding of interprofessional work, and program satisfaction. Although no program-irrelevant variables were entered, the issue of the precise relation between outcome variables and program content remains unsettled.

A strength of this study was the large sample size allowing for multivariate analyses and the inclusion of dropout analyses. Four limitations to this study should also be noted. The most obvious one is that the lack of a randomized waiting list control group prevents firm conclusions about program effects. Second, two biases could have been measured. Health professionals signing up for the program may produce positively skewed attitudinal data with low variability due to initial positive attitudes, and this might be facilitated by the fact that the program coordinators were responsible for the collection of data. Hence, errors of measurements may occur because of a social desirability bias. Still, this was countered in the one-year follow-up by using home-based data collection, which contained the most specific questions about the experienced spin-off effects. The second source of measurement error is an acquiescence bias, appearing due to a large number of items and the unified response format. However, this is countered by the variety of themes covered in the instrument package, as well as the fairly large intervals between data collection sessions. Third, direct measures of attitudes toward eating disorder patients were not included. Finally, the multivariate analyses indicate that possible program benefits in terms of knowledge and attitudes were not fully captured by the present measures.

In conclusion, the evidence supports the expectations of a high level of satisfaction from participating in the program. Initial benefits of participating in the present educational program were sustained, yet longer-term impacts were weaker. The high program satisfaction reported in the current as well as in the previous studies in the field may inspire researchers to pursue causal effects of educational programs using a waiting-list controlled design. In so doing, self-selection of participants should be avoided. This opens for a careful selection procedure by strategic appointments. Such a procedure may provide an opportunity to appoint key personnel related to clinical work as well as the total chain of care in the pursuit of improving clinical services. 
17

Benefits from an Interprofessional Education Program

Rosenvinge \& Pettersen

Journal of Research in Interprofessional Practice and Education

Vol. 6.1

June 2016

\section{References}

1. Rosenvinge, Jan H., Skårderud, Finn, \& Thune-Larsen, Kari-Brith. (2003). Can educational programmes raise clinical competence in treating eating disorders? Results from a Norwegian trial. European Eating Disorders Review, 11, 329-343.

2. Rosenvinge, Jan H., \& Sundgot-Borgen, Jorunn. (1999). Eating disorders - how is the treatment organized? A national survey. Journal of the Norwegian Medical Association, 119, 21-23.

3. Rosenvinge, Jan H., \& Pettersen, Gunn. (2006). Service offered to patients with eating disorders in the Norwegian health care. A national survey. Journal of the Norwegian Psychological Association, 43, 1159-1168.

4. Currin, L., Waller, Glenn, \& Schmidt, Ulrike. (2009). Primary care physicians' knowledge of and attitudes towards eating disorders. Do they affect clinical actions? International Journal of Eating Disorders, 42, 453-458.

5. Hay, Philippa J., De Aangelis, Carlie, Milar, Harry, \& Mond, Jonathan M. (2005). Bulimia nervosa mental health literacy of general practitioners. Primary Care and Community Psychiatry, 10, 103-198.

6. Morgan, John F. (1999). Eating disorders and gyneacology: Knowledge and attitudes among clinicians. Acta Obstetrica et Gynaecologia Scandinavica, 78, 233-239.

7. Fleming, Judith, \& Szmuckler, George I. (1992). Attitudes of medical professionals towards patients with eating disorders. Australian \& New Zealand Journal of Psychiatry, 26, 436-443.

8. Jones, William R., Saedi, Saeideh, \& Morgan, John F. (2013). Knowledge and attitudes of psychiatrists towards eating disorders. European Eating Disorders Review, 21, 84-88.

9. Rodgers, Rachel F., Paxton, Susan, McLean, Sian A., Massey Robin, Mond, Jonathan, Hay, Phillipa, \& Rodgers, Bryan. (2015). Stigmatizing attitudes and beliefs towards bulimia nervosa: The importance of knowledge and eating disorder symptoms. Journal of Nervous and Mental disease, 203, 259-263.

10. Album, Dag, \& Westrin, Steinar. (2008). Do diseases have a prestige hierarchy? A survey among physicians and medical students. Social Sciences and Medicine, 66, 182-188.

11. Rosenvinge, Jan H., Pettersen, Gunn, \& Olstad, Reidun. (2009). The prestige of diseases: A general population study. Open Sociology Journal, 2, 23-29.

12. Reeves, Scott, Tassone, Maria, Parker, Katherine, Wagner, Susan. J, \& Simmons, Brian. (2012). Interprofessional education: An overview of key developments in the past three decades. Work, 41, 233-245.

13. Reeves, Scott, Goldman, Janice, Burton, A, \& Sawatzky-Girling, Brenda. (2010). Synthesis of systematic review evidence of interprofessional education. Journal of Allied Health, 39(suppl 1), 198-203.

14. Fairburn, Christopher G., \& Cooper, Zafra. (2011). Therapist competence, therapy quality and therapist training. Behaviour Research and Therapy, 49, 373-378.

15. Fairburn Christopher G., \& Wilson, G.Terrence. (2013). The dissemination and implementation of psychological treatments: Problems and solutions. International Journal of Eating Disorders, 46, 516-521.

16. Helgadottir, Fjola,, \& Fairburn, Christopher G. (2014). Web-centered training in psychological treatments: A study of therapist preferences. Behaviour Research and Therapy, 52, 61-63.

17. Buhl, Charlotte. (1993). A multiprofessional educational programme on eating disorders for the psychiatric health services in Norway. European Eating Disorders Review, 1, 90-99.

18. DeBate, Rita D., Severson, Herbert H., Cragun, Deborah L., Gau, Jeff M., Merrell, Laura K., Bleck, Jennifer R., Christiansen, Steve, Koerber, Anne, Tomar, Scott L., Tomar, S., Brown, Kelli R, McCormack Brown, R., Tedesco, Lisa, \& Hendricson, William. (2013). Evaluation of a theorydriven e-learning intervention for future oral healthcare providers on secondary prevention of disordered eating behaviors. Health Education Research, 28, 472-484.

19. Linville, Deanna, Aoyama, Tessa, Knoble, Naomi, \& Gau, Jeff. (2012). The effectiveness of a brief eating disorder training programme in medical settings. Journal of Research in Nursing, 18, 544-558.

20. Rosenberg, Marilyn. (2014). Effects of an eating disorders seminar on the knowledge and attitudes of school professionals. Dissertation Abstracts International Section A: Humanities and Social Sciences, 75 .

21. Rosenvinge, Jan H., Silvera, David H., Bergersen, Trine D., Perry, Judy A., Bjørgum, Lars, \& Holte, Arne (2001). A new instrument measuring bulimia nervosa and disturbed eating patterns: Development and validation of a 5-item scale (EDS-5). European Eating Disorders Review, 9, 123-132.

22. Pettersen, Gunn, Rosenvinge, Jan H., Thune-Larsen, Kari-Brith, \& Wynn, Rolf. (2012). Clinical confidence following an interprofessional program on eating disorders for health care professionals: A qualitative analysis. Journal of Multidisciplinary Health Care, 5, 201-205. 